\title{
$P$-Values for non-standard distributions with an application to the DF test
}

\author{
Jerome $\operatorname{Adda}^{\mathrm{a}, \mathrm{b}}$, Jesús Gonzalo ${ }^{\mathrm{b}, *}$ \\ ${ }^{a}$ INRA 63 Boulevard de Brandenbourg, 95205 Ivry sur Seine, France \\ ${ }^{b}$ Department of Economics, Boston University, 270 Bay State Road, Boston, MA 02215, USA
}

Received 14 June 1995; accepted 23 August 1995

\begin{abstract}
The literature on unit roots and structural breaks has produced numerous tests that follow non-standard asymptotic distributions. By fitting a semi-non-parametric model to these distributions, this paper proposes a new simple way to calculate the $p$-values.
\end{abstract}

Keywords: DF Tests; Non-standard distributions; $P$-values; SNP approximations; Unit roots

JEL classification: $\mathrm{C} 12 ; \mathrm{C} 14 ; \mathrm{C} 22$

\section{Introduction}

Most tests for structural breaks and for unit roots follow non-standard asymptotic distributions. These distributions are summarized by reporting a set of critical values (CVs), i.e. $1 \%, 5 \%$ and $10 \%$. With only this information applied researchers cannot calculate the $p$-values and therefore cannot set their own level of significance.

The paper presents a new way to approximate the $p$-values $(\hat{p}(x))$ by using the semi-nonparametric (SNP) techniques introduced by Gallant and Nychka (1987) and Fenton and Gallant (1994). Instead of reporting a set of CVs, we propose a set of parameters that describe a polynomial approximation to the non-standard empirical density. Simple integration of that polynomial produces approximate $p$-values. This can be an efficient way for econometrics software packages to store the empirical distributions of most of the unit root tests and structural break tests.

Previous attempts to approximate $p$-value functions for non-standard test statistics in econometrics were made by Hansen $(1992,1995)^{1}$ and Mackinnon (1994). These works

* Corresponding author. E-mail: jgonzalo@acs.bu.edu.

${ }^{1}$ We received a copy of Hansen (1995) after this research was done. 
basically approximate the $p$-value functions by $H(f(x, \theta))$, where $f(x)$ is a simple polynomial in $x$ with parameters $\theta$, and $H(\cdot)$ is the Normal distribution function in Mackinnon (1994), a transformation of the chi-square distribution in Hansen (1995) and the identity function in Hansen (1992). In Hansen (1992) and Mackinnon (1994) the parameters $\theta$ are estimated by a least squares regression of $H^{-1}(p)$ on a set of quantiles $(x)$. The approach in Hansen (1995) differs in the sense of using an $L^{r}$ norm, for large $r$, to measure the distance $|\hat{p}(x)-p|$.

This paper follows an approach that can be seen as being more general than that in the previous literature, in the sense of not being problem-specific. We do not need to assume any ad hoc $H(\cdot)$ function depending upon whether the test is a unit root test or a structural break test. We can also fit the whole $p$-value function with one model, instead of fitting by regions as in the previous literature.

The paper is organized as follows. Section 2 presents the general framework and methodology. Section 3 shows by a particular example of the DF test for unit roots how to approximate the $p$-values of a non-standard test in practice. Section 4 concludes.

\section{The general framework and methodology}

Our aim is to approximate a $p$-value function $\left(p\left(T_{n}\right)\right)$ that maps an observed test statistic $T_{n}$ into the appropriate value in the range $[0,1]$. This test statistic has an asymptotic distribution $F(x)$ with a corresponding density function $f(x)$. We approximate the $p$-value function $p(x)=F(x)$ by integrating a polynomial approximation of the density function. To do this we simply put the density equal to a Hermite series following the SNP approach introduced by Gallant and Nychka (1987) and Fenton and Gallant (1994). This approach consists of approximating the density function of $T_{n}$ by

$$
f_{n}(x, \theta)=\left[\sum_{i=0}^{k} \theta_{i} w_{i}((x-m) / \sigma)\right]^{2} / \sum_{i=0}^{k} \theta_{i}^{2},
$$

where

$$
\begin{aligned}
& w_{0}(x)=(\sqrt{2 \pi})^{-1 / 2} \mathrm{e}^{-x^{2} / 4} \\
& w_{1}(x)=(\sqrt{2 \pi})^{-1 / 2} x \mathrm{e}^{-x^{2} / 4}, \\
& w_{i}(x)=\left[x w_{i-1}(x)-\sqrt{(i-1)} w_{i-2}(x)\right] / \sqrt{i}
\end{aligned}
$$

The parameters $\theta=\left(m, \sigma, \theta_{i}\right)$ can be estimated by maximum likelihood as in Fenton and Gallant (1994). However, this paper proposes to estimate $\theta$ by minimizing the following loss function:

$$
d_{k}(\theta)=\sum_{j=1}^{q}\left(\left(1 / \alpha_{j}\right)\left(\alpha_{j}-\int_{-\infty}^{c v_{\alpha_{j}}} f_{n}(x, \theta) \mathrm{d} x\right)\right)^{2}
$$


where the first element of $\theta, \theta_{0}$, is set to one and $c v_{\alpha_{j}}$ is the critical value at the $\alpha_{j} \%$ significance level. According to our experience the minimization problem (3) produces better approximations for the $p$-values and converges faster than the equivalent maximum likelihood problem.

The tuning parameters in (3) are

(i) the $q p$-values to match $\left(p_{1} \%, p_{2} \%, \ldots, p_{q} \%\right)$, and

(ii) the number $k$ of parameters $\theta_{i}$.

The next section shows how to choose these tuning parameters in a particular example.

\section{An example: The DF test}

The methodology introduced in Section 2 is valid for obtaining approximate $p$-values of any non-standard distribution. In this section we apply our method to the DF distribution.

Suppose we wish to test the null hypothesis that the variable $y_{t}$ has a unit root. The simplest and most commonly used unit root tests are the DF tests. These tests are based on ordinary least squares estimates of any of the following regressions:

$$
\begin{aligned}
& \mathrm{R}(1): \quad \Delta y_{t}=\lambda y_{t-1}+\sum_{i=1}^{p} \delta_{i} \Delta y_{t-i}+e_{t}, \\
& \mathrm{R}(2): \Delta y_{t}=\mu_{0}+\lambda y_{t-1}+\sum_{i=1}^{p} \delta_{i} \Delta y_{t-i}+e_{t}, \\
& \mathrm{R}(3): \quad \Delta y_{t}=\mu_{0}+\mu_{1} t+\lambda y_{t-1}+\sum_{i=1}^{p} \delta_{i} \Delta y_{t-i}+e_{t},
\end{aligned}
$$

where $\Delta y_{t} \equiv y_{t}-y_{t-1}, t$ is a linear deterministic time trend, $e_{t}$ is an error term, and $\lambda$ is a parameter that equals zero under the null hypothesis of a unit root. The $\mathrm{DF}(i)$ test considered in this paper is the $t$-statistic for $\lambda=0$ in the regression $\mathrm{R}(i)$. This test has a well-known non-standard asymptotic distribution (see Dickey and Fuller, 1979).

In order to calculate the approximated $p$-values of $\operatorname{DF}(i)(i=1,2,3)$ we follow five simple steps:

(i) Calculate the quantiles $(x)$ from the empirical distribution function of the $\operatorname{DF}(i)$ test constructed from 50000 independent draws from their (approximate) asymptotic distributions. In each draw the sample size is set to $n=2000$.

(ii) Standardize $x$ by estimating the mean $(m)$ and the standard deviation $(\sigma)$ by their sample counterparts. This does not change the results but increases the speed of convergence of the minimization problem (3).

(iii) Decide which $q p$-values to match in (3). In this application we have chosen only 16 $p$-values $(0.01,0.02,0.03,0.05,0.06,0.08,0.10,0.12,0.15,0.17,0.20,0.25,0.30,0.40,0.50$, and 0.60$)$. There is a higher concentration in the left tail because of the nature of the DF test.

(iv) Minimize the function $d_{k}(\theta)$ for different values of $k$. Plot $d_{k}\left(\theta_{i}\right)$ on $k$, as in Fig. 1, and choose $k$ accordingly. From Fig. $1, k$ has been set equal to six.

(v) Calculate the approximate $p$-values by simple integration of $f_{n}(x, \theta)$ from $-\infty$ to $c v_{\alpha_{i}}$. 


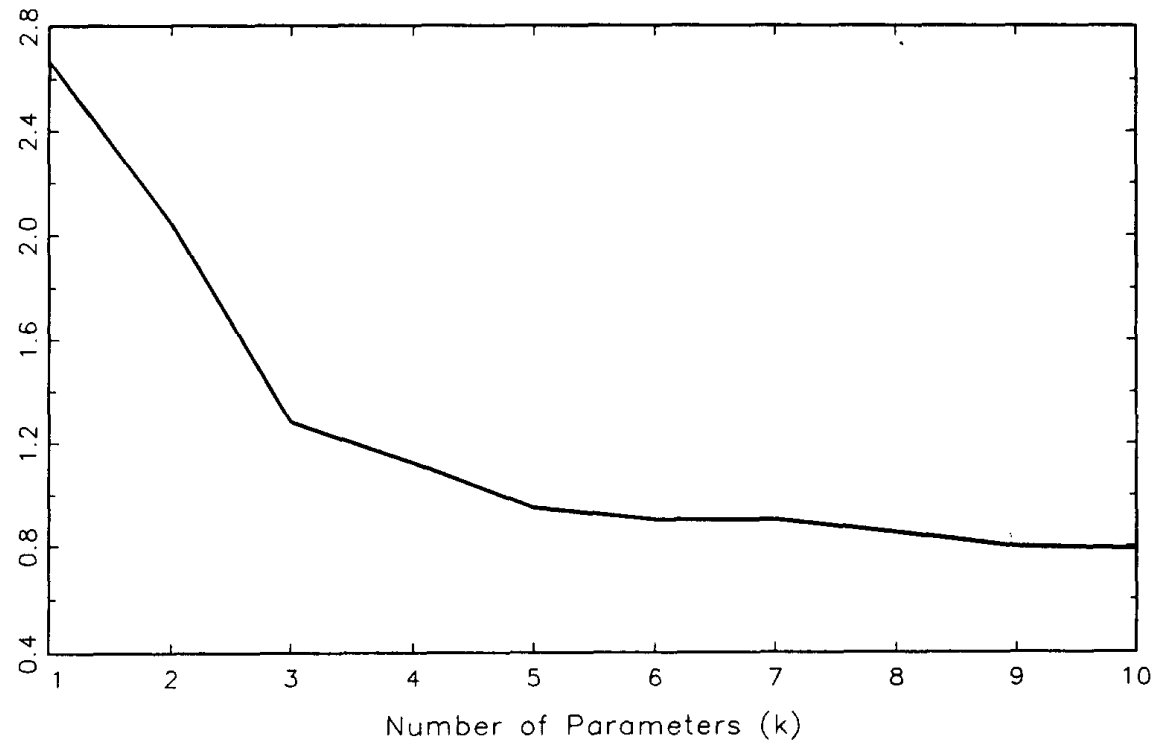

Fig. 1. Log of objective function $\left(d_{k}(\vartheta)\right)$ for the $\mathrm{DF}(3)$ test.

Once the $\theta_{i}$ parameters are estimated, the integration can be done by any standard software (GAUSS, MATHEMATICA, MATLAB, ...).

Table 1 shows the estimated parameters of the polynomial approximation $f_{n}(x, \theta)$ and Table 2 the approximate $p$-values and the absolute error of the approximation. Notice that although

Table 1

Approximate empirical density of the DF $t$-test for $p \leqslant 0.60$

$f_{n}(x, \theta)=\left[\sum_{i=0}^{k} \theta_{i} w_{i}((x-m) / \sigma)\right]^{2} / \sum_{i=0}^{k} \theta_{i}^{2}$
$w_{0}(x)=(\sqrt{2 \pi})^{-1 / 2} \mathrm{e}^{-x^{2} / 4}$
$w_{1}(x)=(\sqrt{2 \pi})^{-1 / 2} x \mathrm{e}^{-x^{2} / 4}$
$w_{i}(x)=\left[x w_{i-1}(x)-\sqrt{i-1} w_{i-2}(x)\right] / \sqrt{i}$

\begin{tabular}{llll}
\hline Parameter & \multicolumn{1}{c}{$\mathrm{DF}(1)$} & $\mathrm{DF}(2)$ & \multicolumn{1}{c}{$\mathrm{DF}(3)$} \\
\hline$m$ & -0.42483 & -1.53206 & -2.17745 \\
$\sigma$ & 0.97505 & 0.83702 & 0.75122 \\
$\theta_{1}$ & -0.30044 & -0.65441 & -0.04769 \\
$\theta_{2}$ & 0.02413 & -0.61767 & -0.09269 \\
$\theta_{3}$ & 0.50388 & -0.23093 & -0.10166 \\
$\theta_{4}$ & 0.51716 & 0.12300 & -0.09983 \\
$\theta_{5}$ & 0.28817 & 0.17911 & -0.06281 \\
$\theta_{6}$ & 0.02567 & 0.12666 & -0.06952 \\
\hline
\end{tabular}


only $16 p$-values are chosen to be matched in (3), the approximation works uniformly well for all the $p$-values from 0.01 to 0.60 . In fact, for the case we focus on in this application (DF(3)), the $\hat{p}(x)$ are very close to the true ones in the whole range $[0,1]$.

Similar results are available for different sample sizes. They are not reported here to keep the paper short.

The calculations have been done using the Optimum library in GAUSS. The programs are available upon request.

Table 2

Approximate $p$-values for the DF $t$-test

\begin{tabular}{|c|c|c|c|c|c|c|}
\hline \multirow[b]{2}{*}{$p$-values $(\%)$} & \multicolumn{3}{|c|}{ Approximate $p$-values } & \multicolumn{3}{|c|}{ Absolute error } \\
\hline & $\mathrm{DF}(1)$ & $\mathrm{DF}(2)$ & $\mathrm{DF}(3)$ & $\mathrm{DF}(1)$ & $\mathrm{DF}(2)$ & $\mathrm{DF}(3)$ \\
\hline 1 & 1.00319 & 0.99984 & 1.00320 & 0.00003 & 0.00000 & 0.00003 \\
\hline 2 & 1.98983 & 2.00171 & 1.99539 & 0.00010 & 0.00002 & 0.00005 \\
\hline 3 & 2.98137 & 2.99925 & 2.95448 & 0.00019 & 0.00001 & 0.00046 \\
\hline 4 & 4.04239 & 3.99781 & 4.07692 & 0.00042 & 0.00002 & 0.00077 \\
\hline 5 & 5.04151 & 4.96833 & 5.09336 & 0.00042 & 0.00032 & 0.00093 \\
\hline 6 & 6.02473 & 6.05821 & 6.05094 & 0.00025 & 0.00058 & 0.00051 \\
\hline 7 & 7.01476 & 6.99862 & 7.06335 & 0.00015 & 0.00001 & 0.00063 \\
\hline 8 & 7.99055 & 7.96124 & 7.96878 & 0.00009 & 0.00039 & 0.00031 \\
\hline 9 & 9.01392 & 8.94637 & 8.93215 & 0.00014 & 0.00054 & 0.00068 \\
\hline 10 & 10.02747 & 9.97407 & 9.94182 & 0.00027 & 0.00026 & 0.00058 \\
\hline 11 & 11.00684 & 11.08123 & 10.92839 & 0.00007 & 0.00081 & 0.00072 \\
\hline 12 & 11.97744 & 12.05579 & 11.96005 & 0.00023 & 0.00056 & 0.00040 \\
\hline 13 & 12.98543 & 13.05882 & 12.99713 & 0.00015 & 0.00059 & 0.00003 \\
\hline 14 & 13.99103 & 14.04091 & 13.99807 & 0.00009 & 0.00041 & 0.00002 \\
\hline 15 & 14.98372 & 15.05265 & 14.93359 & 0.00016 & 0.00053 & 0.00066 \\
\hline 16 & 15.97357 & 15.93928 & 15.95400 & 0.00026 & 0.00061 & 0.00046 \\
\hline 17 & 16.94230 & 16.90459 & 16.93799 & 0.00058 & 0.00095 & 0.00062 \\
\hline 18 & 17.96399 & 17.94639 & 17.98578 & 0.00036 & 0.00054 & 0.00014 \\
\hline 19 & 18.96067 & 18.96677 & 19.02162 & 0.00039 & 0.00033 & 0.00022 \\
\hline 20 & 19.97044 & 20.00598 & 20.07539 & 0.00030 & 0.00006 & 0.00075 \\
\hline 21 & 21.01644 & 21.01417 & 21.05782 & 0.00016 & 0.00014 & 0.00058 \\
\hline 22 & 22.06442 & 21.99547 & 22.07475 & 0.00064 & 0.00005 & 0.00075 \\
\hline 23 & 23.03593 & 22.97540 & 23.03562 & 0.00036 & 0.00025 & 0.00036 \\
\hline 24 & 24.00016 & 24.00336 & 24.06584 & 0.00000 & 0.00003 & 0.00066 \\
\hline 25 & 24.98652 & 25.02584 & 25.05570 & 0.00013 & 0.00026 & 0.00056 \\
\hline 30 & 29.98828 & 29.97709 & 30.03994 & 0.00012 & 0.00023 & 0.00040 \\
\hline 35 & 35.02969 & 34.99348 & 34.96467 & 0.00030 & 0.00007 & 0.00035 \\
\hline 40 & 40.03144 & 39.99644 & 40.01450 & 0.00031 & 0.00004 & 0.00015 \\
\hline 45 & 45.03378 & 44.88271 & 45.08477 & 0.00034 & 0.00117 & 0.00085 \\
\hline 50 & 50.23323 & 50.03430 & 50.11525 & 0.00233 & 0.00034 & 0.00115 \\
\hline 55 & 55.25007 & 55.08260 & 54.96449 & 0.00250 & 0.00083 & 0.00036 \\
\hline 60 & 59.83218 & 59.98085 & 59.83991 & 0.00168 & 0.00019 & 0.00160 \\
\hline 65 & 63.89082 & 64.84299 & 64.91459 & 0.01109 & 0.00157 & 0.00085 \\
\hline 70 & 67,10778 & 69.25286 & 69.65490 & 0.02892 & 0.00747 & 0.00345 \\
\hline
\end{tabular}




\section{Conclusion}

This paper shows a simple and general way to approximate the $p$-values of non-standard distributions.

\section{References}

Dickey, D.A. and W.A. Fuller, 1979, Distribution of the estimators for autoregressive time series with a unit root, Journal of the American Statistical Association 74, 427-431.

Fenton, V.M. and R.A. Gallant, 1994, Qualitative and asymptotic performance of SNP density estimator, Mimeo, University of North Carolina.

Gallant, R.A. and D.W. Nychka, 1987, Seminonparametric maximum likelihood estimation, Econometrica 55, 363-390.

Hansen, B.E., 1992, Test for parameter instability in regressions with I(1) processes, Journal of Business and Economic Statistics 10, 321-335.

Hansen, B.E., 1995, Approximate asymptotic $p$-values for structural change tests, Mimeo, Boston College.

Mackinnon, J.G., 1994, Approximate asymptotic distribution functions for unit-root and cointegration tests, Journal of Business and Economic Statistics 12, 167-176. 